\title{
Asociación de la densidad calórica de la leche materna según parámetros antropométricos de las madres y sus hijos
}

\author{
Astrid Bruno-Huamán1,2,a, Solange Valdivia-Lívano1,2,a, Christian R. Mejia 1,3,b. \\ ${ }^{1}$ Escuela Académico Profesional de Medicina Humana, Universidad Continental. Huancayo, Junín, Perú. ${ }^{2}$ Sociedad \\ Científica Médico Estudiantil Continental - SOCIMEC. Huancayo, Junín, Perú. ${ }^{3}$ Asociación Médica de Investigación y \\ Servicios en Salud, Lima, Perú.
}

$\mathrm{a}_{\text {Estudiante de medicina. }}{ }^{\mathrm{b}}$ Médico-cirujano.

\section{RESUMEN}

Antecedentes: La lactancia materna es indispensable para el desarrollo del lactante, y ésta cambia según varios factores, entre los cuales está el estado nutricional de la madre. Objetivo: Determinar si existe asociación entre la densidad calórica de la leche materna y las medidas antropométricas de la madre y su lactante Método: Se realizó un estudio transversal-analítico, que incluyó a madres y a sus lactantes de 1-6 meses de edad, en el Hospital Materno Infantil "El Carmen"-Huancayo, Perú. Se extrajo leche de las madres participantes según normas internacionales. Se obtuvieron las medidas antropométricas de las madres y sus lactantes. El aporte calórico de la leche se midió con la fórmula de Lucas. Se usó estadística descriptiva y analítica, con intervalos de confianza del 95\% y el valor $p<0,05$ como estadísticamente significativo. Resultados: De las 51 encuestadas, la media del contenido calórico fue de 64,5 kcal/100ml. En el análisis multivariado, ajustado por la edad del lactante, el contenido calórico era mayor en los niños de peso normal en comparación de los desnutridos (peso/edad:+9,9, $p<0,001$; talla/edad:+8,5, $p=0,018$ y peso/talla:+7,1, p=0,006); asimismo, en el peso/edad el contenido calórico de la leche materna de los niños obesos era menor que de los desnutridos $(-14,3, p<0,001)$. Conclusión: Las variaciones del contenido calórico de la leche fueron diferentes según los tres parámetros de los niños eutróficos.

\section{PALABRAS CLAVE: Leche humana, lactancia, lactancia materna, nutrición materna, lactante}

\section{SUMMARY}

Background: Breastfeeding is essential for the proper development of the infant, and it changes depending on factors such as the nutritional status of the mother. Aim: To determine the association between caloric density of human milk and anthropometric measurements of the mother and infant. Method: A cross-analytical study; involving mothers and infants 1-6 months of age in the Maternity Hospital "El Carmen"-Huancayo, Peru. Participating mother's milk was extracted according to international standards. Anthropometric measurements of mothers and their infants were obtained. The caloric intake of milk was measured with the Lucas technique. We use analytical and descriptive statistics, confidence intervals at $95 \%$ and $p<0.05$ as statistically significant value. Results: Of the 51 respondents, the average heat content was $64.5 \mathrm{kcal} / 100 \mathrm{ml}$. In multivariate analysis, adjusted for infant age, caloric content was higher in children of normal weight compared malnourished (weight/age: $+9.9, p<0.001$; size/age: $+8.5, p=0.018$ and weight/size: $+7.1, p=0.006$ ) Also in the weight/age caloric content of breast milk of obese children it was lower than that of malnourished $(-14.3, p<0.001)$. Conclusion: Variations of the caloric content of milk differed among the three parameters of eutrophic children.

KEY WORDS: Human milk, lactation, breast feeding, maternal nutrition, infant 


\section{INTRODUCCIÓN}

La alimentación durante los primeros seis meses de vida debe ser exclusivamente con leche materna, a partir de ese periodo con alimentos complementarios, siendo este el estándar normativo para la alimentación infantil y la nutrición, propuesta por la Organización Mundial de Salud (1), ya que la lactancia materna (LM) es una fuente exclusiva de nutrientes durante los primeros meses de vida y primordial para el desarrollo físico y mental del lactante (2).

Se sabe que la composición de la leche humana es dinámica y variable, que difiere de la alimentación con fórmula infantil, que esta estandarizada en su composición (1). Esto se basa en que la leche, al ser un fluido vivo, tiene variaciones a las distintas horas del día, entre cada mamada, en una misma mamada, según la temperatura del ambiente, estado nutricional de la madre y en muchas otras circunstancias (3-4).

Si la leche materna no es adecuada, puede traer problema en la nutrición del niño, como son la desnutrición y la obesidad, que son consecuencia de la LM sub-óptima, siendo esto considerado como un factor de riesgo de desnutrición y mortalidad durante los primeros dos años de vida (5).

La LM sub-óptima puede ser un factor de riesgo de obesidad en los niños, puesto que al no cubrir los requerimientos calóricos diarios del lactante este tendrá que incluir una alimentación complementaria precoz (antes de los 4 meses) (6). Un meta-análisis mostró que la LM reduce significativamente el riesgo de obesidad en la niñez, aunque depende de la duración (7).

Los mecanismos fisiológicos que toman a la LM como factor protector contra la obesidad infantil y posteriormente contra el síndrome metabólico en la adolescencia, necesitan mayor investigación para que puedan ser explicados, pero ya se cuenta con evidencia científica que avala esa asociación $(8,9)$. En Perú no se han encontrado investigaciones sobre este tema, a pesar de la importancia de la lactancia materna para prevenir la desnutrición y la mortalidad de los niños (5). Además, este tema es una de las prioridades de investigación en la Región Junín, Perú (10) y el $4^{\circ}$ Objetivo de Desarrollo del Milenio (3).

El objetivo de esta investigación fue determinar si existe asociación entre la densidad calórica de la leche materna y las variables antropométricas de la madre y su lactante.

\section{SUJETOSY MÉTODOS}

Se realizó un estudio analítico, transversal, prospectivo y observacional. Se incluyó a madres sin antecedentes de enfermedad independientemente del estado nutricional, mayores de edad, que acudieron al Servicio de Crecimiento y Desarrollo (CRED) del Hospital Materno Infantil "El Carmen"Huancayo, Perú, con hijos sanos al momento de la evaluación y sin antecedentes de enfermedad entre 1 y 6 meses de edad, que daban como único alimento leche materna. Se excluyó a madres que tenían alguna contraindicación para LM por tener alguna patología mamaria que dificulte la lactancia, parto múltiple, hijos de pre-término o pos-término, y/o complicaciones neonatales, y niños con malformaciones congénitas (2 excluidas en total).

Se obtuvo un mínimo tamaño muestral de 44, con un error alfa de 0,05 , error beta de 0,1 y para detectar una diferencia de medias de 1,0; a esto se sumó un $10 \%$ por la tasa de rechazo, obteniendo un tamaño mínimo muestral de 49 , estimado mediante el programa estadístico EPIDAT 4,0. Se realizó el muestreo aleatorio, habiendo encuestado a 51 madres con sus respectivas muestras lácteas.

En la primera fase del estudio se obtuvo la aprobación del proyecto por el Comité de Ética (oficio 259-OADI-HONADOMANI.SB-2015) y la obtención de los permisos por la institución de salud. Luego se entrevistó a las madres previa obtención del consentimiento verbal. La información fue obtenida por contacto directo con solo una de las investigadoras del estudio, quien tomó la muestra de la leche y los datos de la madre de la tarjeta de control y crecimiento del lactante. Esto se realizó en un ambiente privado que proporcionó el hospital, para respetar la privacidad de las madres.

La obtención de la muestra fue por extracción manual supervisada por las obstetrices de la institución, según técnicas recomendadas a nivel internacional. Por ser la leche materna un fluido dinámico que varía según la hora del día, entre cada mamada y en una misma mamada, para la investigación, se consideraron los siguientes criterios: la extracción de la muestra se realizó en horas de la mañana (8:00-10:00 de la mañana) y con un espacio de 3-4 horas desde la última mamada. Se extrajeron $5 \mathrm{ml}$ de leche materna, luego de la obtención se trasladó en un envase aséptico y a una temperatura controlada hasta el laboratorio para su posterior análisis mediante la técnica de Lucas. Se obtuvo el crematocrito y mediante la siguiente fórmula las calorías: Energía $(\mathrm{kcal} / 100 \mathrm{ml})=290+$ $(66,8 \times$ crematocrito (\%)) (11).

De cada alícuota se extrajo un capilar, se selló en un extremo y se centrifugó durante 5 minutos a 12.000 rpm en una micro-centrífuga para hematocrito. Se midió con una regla milimetrada, las columnas de crema y suero (11). De esta manera se obtuvo el crematocrito y el contenido calórico; el crematorito fue el porcentaje de crema con respecto a la longitud total de la columna.

Las variables maternas consideradas en la ficha de recolección de datos fueron: la edad (en años cumplidos), el peso actual (tomado en 
kilogramos con los equipos calibrados del hospital), la talla (en metros y con un tallímetro estándar) y el IMC categorizado en malnutrición $(\mathrm{IMC}<18,5)$, normal (IMC 18,5-24,9) y exceso de peso (IMC $\geq 25$ ). Las variables del lactante fueron: sexo, edad actual (en meses cumplidos), peso al nacer (de la ficha de control), peso actual (tomado en kilogramos con una balanza neonatal calibrada) y talla (tomado en $\mathrm{cm}$ y obtenida con una cinta métrica, según recomendaciones internacionales). Con estas medidas se obtuvieron los parámetros de crecimiento del peso/edad, talla/edad y peso/ talla, considerando las categorías de desnutridos severos, desnutridos moderados, normales y obesidad, para cada parámetro, según lo establece la OMS; siendo categorizados en tres categorías para el análisis bivariado y multivariado: desnutridos (desnutridos severos y moderados), normales y con obesidad.

Los datos se procesaron en el programa Microsoft Excel para Windows 2010, luego se analizaron en el programa estadístico Stata versión 11,1 (Stata Corp LP, College Station, TX, USA). Previa a la descripción de las variables cuantitativas se ejecutó la prueba de normalidad Shapiro-Wilk, siendo descritas con las medidas de tendencia central y dispersión más adecuadas. Las variables categóricas fueron descritas con frecuencias y porcentajes. Para la estadística analítica se trabajó con una significancia estadística del 95\%. Para el análisis bivariado del contenido calórico de la leche materna (cuantitativa) según los parámetros de crecimiento (categórica politómica), las variables maternas (cuantitativas) y del recién nacido (cuantitativas y politómicas) se usó los modelos lineales generalizados, con familia Gaussian, función de enlace identity y ajuste para muestras pequeñas según la edad de los niños; obteniendo el valor $p$, los coeficiente y sus intervalos de confianza al $95 \%$ (IC95\%). Se consideró estadísticamente significativo un valor $p<0,05$.

\section{RESULTADOS}

De las 51 encuestadas, la media de edades fue $26,6 \pm 5,8$ años, el $62,8 \%$ (32) de los lactantes fueron varones, las variables fisio-antropométricas de los niños y las madres se muestran en la Tabla I.

Según los parámetros de crecimiento de los niños (peso/edad, talla/edad y peso/talla), el gran porcentaje de los niños estuvo clasificado como eutrófico (Figura $1 \mathrm{~A}$ ), seguidos del $35,3 \%$ de obesos según la talla para la edad y $15,7 \%$ de peso para la talla. Asimismo, se encontró que el promedio de contenido calórico de la leche materna (Figura 1B) tuvo variación según si los niños fueron desnutridos severos, desnutridos moderados, normales o con obesidad; siendo estadísticamente significativas (análisis bivariado mediante modelos lineales generalizados) cuando se comparó a los niños de peso normal versus los desnutridos severos según el peso/edad $(p=0,001)$, además, de los de peso normal $(p=0,022)$ y obesos $(p=0,032)$ según los desnutridos severo mediante la talla/edad (Figura 1 ).

Tabla I

\section{MEDIDAS FISIO-ANTROPOMÉTRICAS DE LAS MADRES Y SUS NIÑOS EN LA CIUDAD DE HUANCAYO}

\begin{tabular}{|c|c|c|c|c|}
\hline Variable & Media & $\pm \mathrm{DE}$ & Mediana & Rango \\
\hline \multicolumn{5}{|l|}{ De la madre: } \\
\hline Edad (años) & 26,6 & 5,8 & 28,0 & $18-42$ \\
\hline Peso (kg) & 57,6 & 8,8 & 57,2 & $39,3-83,7$ \\
\hline Talla $(\mathrm{m})$ & 1,53 & 0,1 & 1,5 & $1,41-1,67$ \\
\hline Índice de masa corporal $\left(\mathrm{kg} / \mathrm{m}^{2}\right)$ & 24,6 & 3,2 & 24,7 & $17,1-32,7$ \\
\hline Crematocrito (\%) & 5,3 & 2,5 & 5,0 & $2-15$ \\
\hline Contenido calórico de la leche ${ }^{\star}$ & 64,5 & 16,8 & 62,4 & $42,4-129,2$ \\
\hline \multicolumn{5}{|l|}{ Del niño: } \\
\hline Peso al nacer $(\mathrm{kg})$ & 3,0 & 0,6 & 3,1 & $1,5-4,3$ \\
\hline Edad actual (meses) ${ }^{\star}$ & 2,7 & 1,6 & 2,0 & $1-6$ \\
\hline Peso actual (kg) & 5,4 & 1,4 & 5,3 & $2,6-8,9$ \\
\hline Talla actual $(\mathrm{cm})^{\star}$ & 57,2 & 5,9 & 57,8 & $45-78$ \\
\hline
\end{tabular}

DE: Desviación estándar. *Variables no normales. 

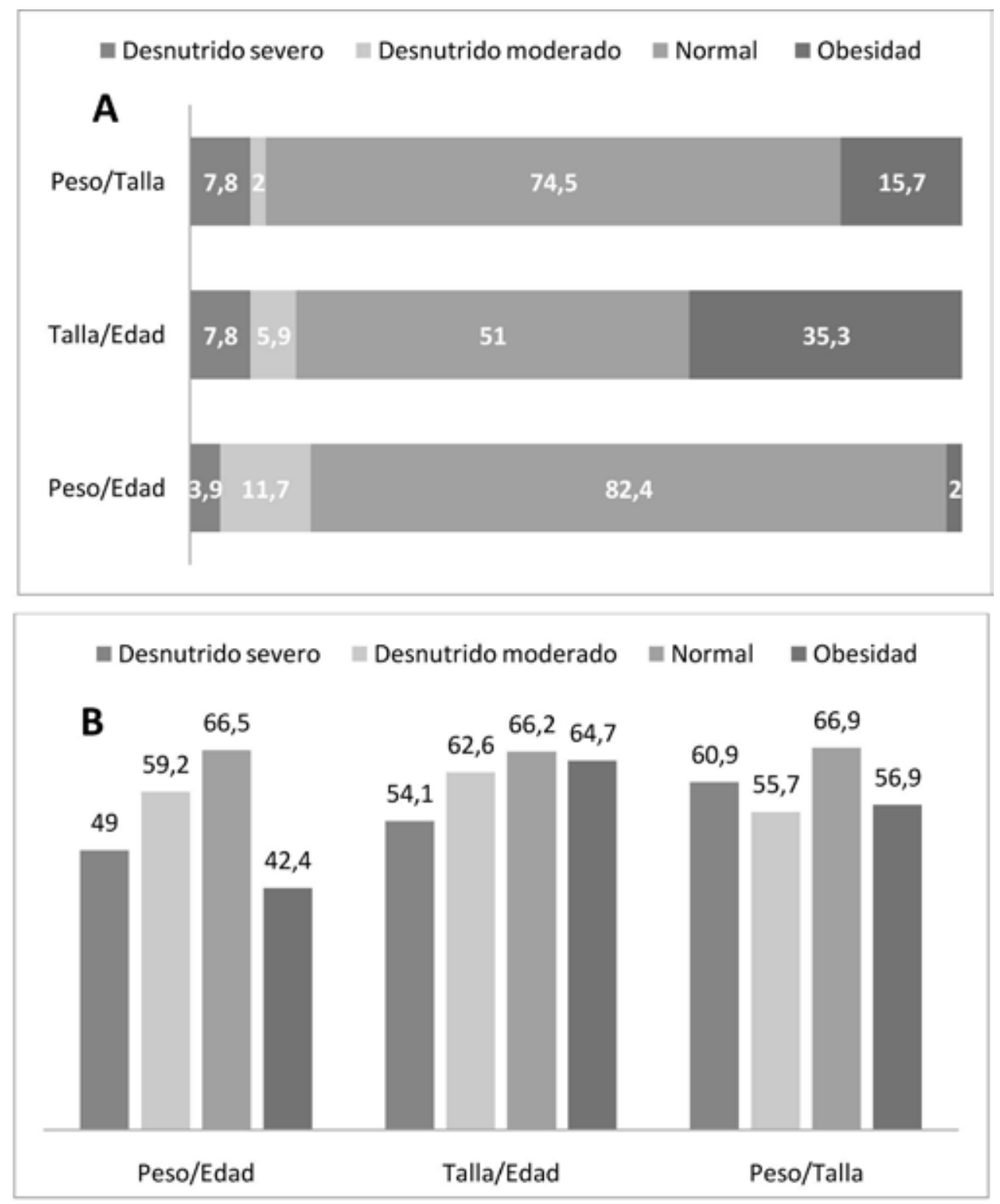

Figura 1. Porcentajes (1A) y el promedio de contenido calórico de la leche materna (1B) según si los niños fueron desnutridos severos, desnutridos moderados, normales o con obesidad.

Al realizar el análisis multivariado según los parámetros de crecimiento de los niños (ajustando por la edad de los niños), se encontró que el contenido calórico de la leche materna de los niños era mayor en los que tenían peso normal a comparación de los que tenían desnutrición (peso/edad: $+9,9$, $\mathrm{p}<0,001$; talla/edad: $+8,5, \mathrm{p}<0,018$ y peso/talla: $+7,1$, $p<0,006$ ); asimismo, en el parámetro de peso/edad el contenido calórico de la leche materna de los niños con obesidad era menor que los que tenían desnutrición $(-14,3, p<0,001)$ (Tabla II). 


\section{Tabla II}

\section{ASOCIACIÓN ENTRE EL CONTENIDO CALÓRICO DE LA LECHE MATERNA Y LAS VARIABLES} DE LAS MADRES Y SUS NIÑOS EN LA CIUDAD DE HUANCAYO

\begin{tabular}{|c|c|c|c|}
\hline Variable & Coeficiente & IC95\% & Valor $p$ \\
\hline \multicolumn{4}{|l|}{ De la madre: } \\
\hline Edad (años) & 0,11 & $-0,46 / 0,68$ & 0,705 \\
\hline Peso (kg) & 0,08 & $-0,45 / 0,61$ & 0,768 \\
\hline Talla (m) & 12,44 & $-47,49 / 72,36$ & 0,684 \\
\hline Índice de masa corporal (kg/m2) & 0,13 & $-1,35 / 1,61$ & 0,863 \\
\hline Crematocrito (\%) & 6,70 & $6,67 / 6,72$ & $<0,001$ \\
\hline \multicolumn{4}{|l|}{ Del niño (ajustado por la edad): } \\
\hline Peso actual (kg) & $<0,01$ & $-0,002 / 0,003$ & 0,738 \\
\hline Talla actual (cm) & 0,27 & $-0,11 / 0,65$ & 0,166 \\
\hline \multicolumn{4}{|l|}{ Peso/Edad: } \\
\hline Desnutrido & \multicolumn{3}{|c|}{ Categoría de comparación } \\
\hline Normal & 9,90 & $5,59 / 14,20$ & $<0,001$ \\
\hline Obesidad & $-14,29$ & $-19,29 /-9,28$ & $<0,001$ \\
\hline \multicolumn{4}{|l|}{ Talla/Edad: } \\
\hline Normal & 8,46 & $1,48 / 15,43$ & 0,018 \\
\hline Obesidad & 7,01 & $-3,08 / 17,82$ & 0,204 \\
\hline \multicolumn{4}{|l|}{ Peso/Talla: } \\
\hline Desnutrido & \multicolumn{3}{|c|}{ Categoría de comparación } \\
\hline Normal & 7,05 & $2,07 / 12,03$ & 0,006 \\
\hline Obesidad & $-3,88$ & $-13,36 / 5,60$ & 0,422 \\
\hline
\end{tabular}

Coeficiente y valor $\mathrm{p}$ obtenidos con modelos lineales generalizados, con familia Gaussian, función de enlace identity y ajuste para muestras pequeñas.

\section{DISCUSIÓN}

Según los resultados encontrados el contenido calórico de la leche materna se asocia a los parámetros de crecimiento de los niños, siendo diferente en los tres parámetros antropométricos de niños con peso normal en comparación de los desnutridos, independientemente del estado nutricional de la madre.

La leche humana es reconocida como la alimentación óptima de todos los lactantes, debido a sus beneficios para la salud a los recién nacidos y sus madres. Un sinnúmero de organizaciones recomiendan la lactancia materna exclusiva durante los primeros seis meses de vida (12-14). La composición de la leche humana es compleja, que proporcionan la energía necesaria para el infante y siendo el alimento ideal (15). La composición de este fluido destaca por su variabilidad (16), sin embargo, puede haber factores que condicionen una variación en la composición y la calidad de la misma, condicionando una lactancia sub-óptima.

El contenido energético promedio de la leche materna madura fue de $64,5 \mathrm{kcal} / 100 \mathrm{ml}$, esta cifra se encuentra dentro de los límites normales descritos por la bibliografía $60-70 \mathrm{kcal} / 100 \mathrm{ml}$ (17). Además, se encontró que hubo diferencias del contenido calórico de las madres según el estado nutricional de sus niños, siendo mucho menor en las madres de los niños que tuvieron desnutrición severa; pudiendo ser esta asociación generada por un aporte insuficiente de nutrientes, el contenido hipocalórico de la leche y la desnutrición severa también estuvo asociado. Todo esto se podría estar ocasionando debido a que no se otorgan por completo los beneficios que ofrece la lactancia materna, caso similar a cuando se usan las fórmulas artificiales (18).

También se encontró que el contenido calórico de la leche materna de los niños que tenían un 
adecuado peso era mejor que los que tenían desnutrición, lo que es respaldado por una investigación que evaluó a los lactantes por su estado nutricional, evidenciando que del total de niños que se alimentaban de leche materna exclusiva, la gran mayoría resultaron con peso considerado como normal (19). En otra investigación la evolución de los recién nacidos malnutridos fue muy buena al darles lactancia materna exclusiva por madres con medidas antropométricas adecuadas, ya que lograron mejores índices de crecimiento (20). Los pacientes eutróficos de otra investigación, recibieron lactancia materna exclusiva por 6 meses; contrariamente, más de la mitad de los pacientes malnutridos por exceso o por defecto, abandonaron la lactancia materna exclusiva antes de ese período (21). Todo esto ratifica que una adecuada lactancia exclusiva hasta el sexto mes de vida favorece un adecuado crecimiento y desarrollo del niño (22).

Por último, resaltamos que los niños que tenían obesidad recibían un menor contenido calórico de la leche materna, esto se relaciona con estudios que describen que cuanto más prolongada sea la lactancia materna mayor protección contra la obesidad infantil (23), pero dichos estudios no evaluaron la calidad de la leche materna, que si fue motivo de nuestro estudio, en donde encontramos que el contenido calórico de la leche que estos niños recibían era incluso más hipocalórica con respecto a la leche que recibían los niños desnutridos. Esto puede deberse a diversos motivos que influyan para que la leche haya tenido una distinta composición energética (24).

Se tuvo la posible limitación del sesgo de información, ya que por la memoria de las madres algunos datos pudieron no ser exactos, sin embargo se cree que esto fue mínimo, ya que se preguntó por hecho que no fueron hace mucho tiempo.

\section{CONCLUSIÓN}

Según los resultados encontrados se concluye que el contenido calórico de la leche materna se asocia a los parámetros de crecimiento de los niños, siendo diferente en los tres parámetros de los niños con peso normal a comparación de los desnutridos. Se recomiendan hacer estudios longitudinales que evalúen de manera prospectiva si el contenido calórico de las madres influirá en la nutrición de los niños en los siguientes meses.

AGRADECIMIENTOS: Queremos agradecer a la Sociedad Científica Médico Estudiantil Continental (SOCIMEC) por habernos dado a conocer la importancia de la investigación en el desarrollo profesional y en el de las ciencias médicas. A nuestros maestros, Dr. Gerardo Rios Cerna, Dr. Julio Troncoso Mena y Dra. Obs. Maria Romero Santillana, por la orientación y motivación para continuar en este reto, además, a nuestras compañeras Geraldine, Mayra, Yanina y Leticia por su importante apoyo en la recolección de muestras, aporte en ideas y organización de la información. Este artículo fue preparado como parte de las actividades conjuntas del sexto Grupo de Investigación de las SOCEM's (GIS) y la Asociación Médica de Investigación y Servicios en Salud (AMISS). Este manuscrito fue preparado por Astrid Bruno-Huamán, en cumplimiento del requisito de participación del sexto GIS, Lima, Perú.

\section{REFERENCIAS}

1. Chung MY. Factors affecting human milk composition. Pediatr Neonatol 2014;55(6):421-2.

2. Garc R. Composición e inmunología de la leche humana. Acta Pediátrica México 2011;32(4):223-30.

3. Juez García G, Niño Moya R, Ortega Weason R, Mena Nannig P, Santander Rigollete S, González Opazo M. Lactancia Materna. 2da ed. Juez García G, Niño Moya R, Ortega Weason R, Mena Nannig P, Santander Rigollete S, González Opazo M, editors. Chile; 2010.

4. Álvarez de Acosta T, Rossell-Pineda M, Cluet de Rodriguez I, Fuenmayor E. Macronutrientes en leche de madres desnutridas. Arch Latinoam Nutr 2009;59(5):159-65.

5. Black RE, Victora CG, Walker SP, Bhutta ZA, Christian $\mathrm{P}$, de Onis $\mathrm{M}$, et al. Maternal and child undernutrition and overweight in low-income and middle-income countries. Lancet 2013;382(9890):427-51.

6. Hunsberger M; IDEFICS Consortium. Early feeding practices and family structure: associations with overweight in children. Proc Nutr Soc 2014;73(1):1326.

7. Arenz S, Rückerl R, Koletzko B, von Kries R. Breastfeeding and child hood obesity-A systematic review. Int J Obes Relat Metab Disord 2004;28(10):1247-56.

8. Butte NF. Impact of infant feeding practices on child hood obesity. J Nutr 2009;139(2):412S-6S.

9. Novaes JF, Lamounier JA, Colosimo EA, Franceschini SC, Priore SE. Breastfeeding and obesity in Brazilian children. Eur J Public Health 2012; 22(3):383-9.

10. Dirección Ejecutiva de Vigilancia Alimentaria y Nutricional. Centro Nacional de Alimentación y Nutrición. Instituto Nacional de Salud. Situación Nutricional del Perú 2006-2013. Disponible en: http:// www.ins.gob.pe/repositorioaps/0/5/jer/situ_vigi_cenan/Situaci\%C3\%B3n\%20nutricional\%20en\%20 ni \% C 3 \% B 1 os \% 20 menores $\% 20$ de $\% 205 \% 20$ a\%C3\%B1os\%202006-2013.pdf

11. Lucas A, Gibbs JA, Lyster RL, Baum JD. Creamatocrit: simple clinical technique for estimating fat concentration and energy value of human milk. Br Med J 1978;1(6119):1018-20.

12. WHO. Global strategy for infant and young child feeding [Internet]. World Health Organization; 2003. pp 30. Disponible en: http://www.who.int/nutrition/publications/infantfeeding/9241562218/en/

13. Statement $P$. Breastfeeding and the use of human milk. Pediatrics 2012;129(3):e827-41.

14. Force USPST. Clinical guidelines. Clin Guidel 1995;310(8):670.

15. Mennella JA. Mother's milk: a medium for early flavor experiences. J Hum Lact 1995;11(1):39-45.

16. Neville MC, Keller RP, Seacat J, Casey CE, Allen JC, Archer P. Studies on human lactation. I. Within-feed 
and between-breast variation in selected components of human milk. Am J Clin Nutr 1984;40:635-46.

17. Martinez de Victoria E, Maldonado J. Nutrición Humana en el Estado de Salud. 5ta ed. España: Ed. Médica Panamericana; 2010.

18. Díaz MG, Villares JMM, Serra JD. Y ahora, si es preciso aumentar el contenido en energía y nutrientes en la alimentación de un lactante, ¿qué debo emplear? Acta Pediatr Esp 2014;72(9):195-201.

19. Calzado Mustelier M, Rodríguez Rivero L, Vargas Fajardo E, Vistel Sánchez M. Influencia de la lactancia materna en la salud del niño. Rev Cubana Enf 2000;16(2):122-7.

20. Díaz-Argüelles Ramírez-Corría V. Lactancia materna: evaluación nutricional en el recién nacido. Rev Cubana Pediatr 2005;77(2). Disponible en: http://scielo.sld. cu/pdf/ped/v77n2/ped05205.pdf

21. Basain Valdés JM, Pacheco Díaz LC, Valdés Alonso M del C, Miyar Pieiga E, Maturell Batista A. Duración de lactancia materna exclusiva, estado nutricional y dislipidemia en pacientes pediátricos. Rev Cubana Pediatr 2015;87(2):156-66.

22. Jiménez Acosta S, Sánchez Ramos R PPS. Guías Alimentarias para niñas y niños cubanos hasta dos años de edad. INHA. La Habana; 2012;21(1):1-2.

23. Basain J, Pacheco L, Valdés M, Miyar E, Maturell A. Duración de lactancia materna exclusiva, estado nutricional y dislipidemia en pacientes pediátricos. Rev Cubana Pediatr 2015;87(2). Disponible en: http:// www.bvs.sld.cu/revistas/ped/vol87_2_15/ped03215. htm.

24. Shellhorn C, Valdés V. La leche materna, composición, beneficios y comparación con la leche de vaca. Manual de Lactancia Materna para Profesionales de la Salud. 1995;30. Disponible en: http://www.unicef.cl/ lactancia/docs/mod01/Mod\%201beneficios\%20manual.pdf. 